\title{
Exponential mixing for finite-dimensional approximations of the Schrödinger equation with multiplicative noise
}

\author{
Vahagn Nersesyan
}

Communicated by Armen Shirikyan, received June 5, 2008.

\begin{abstract}
We study the ergodicity of finite-dimensional approximations of the Schrödinger equation. The system is driven by a multiplicative scalar noise. Under general assumptions over the distribution of the noise, we show that the system has a unique stationary measure $\mu$ on the unit sphere $S$ in $\mathbb{C}^{n}$, and $\mu$ is absolutely continuous with respect to the Riemannian volume on $S$. Moreover, for any initial condition in $S$, the solution converges exponentially fast to the measure $\mu$ in the variational norm.
\end{abstract}

\section{Contents}

1. Introduction 167

2. Main result 169

3. Controllability results 174

4. Proof of Lemmas 2.4 and $2.5 \quad 178$

5. Application 181

6. Appendix 181

References 182

\section{Introduction}

We consider the problem

$$
\begin{aligned}
i \frac{\mathrm{d} z}{\mathrm{~d} t} & =\Lambda z+\beta(t) B z+\varepsilon F(z), \\
z(0) & =z_{0},
\end{aligned}
$$

1991 Mathematics Subject Classification. 34, 37, 35.

Key words and phrases. Exponential mixing, ergodicity, Schrödinger equation, multiplicative noise.

(c)2009 International Press 
where $\Lambda$ and $B$ are Hermitian matrices, $F: \mathbb{C}^{n} \rightarrow \mathbb{C}^{n}$ is a real-analytic function such that the scalar product $\langle F(z), z\rangle$ is real for any $z \in \mathbb{C}^{n}$, and $\varepsilon \in \mathbb{R}$ is a small constant. We assume that $\beta(t)$ is a random process of the form

$$
\beta(t)=\sum_{k=0}^{+\infty} I_{k}(t) \eta_{k}(t-k), \quad t \geq 0
$$

where $I_{k}(\cdot)$ is the indicator function of the interval $[k, k+1)$ and $\eta_{k}$ are independent identically distributed (i.i.d.) random variables in $L^{2}([0,1], \mathbb{R})$.

The restriction of the solution of (1.1), (1.2) at integer times formes a Markov chain. The aim of this paper is the study of ergodicity of this chain. Noting that the unit sphere $S$ in $\mathbb{C}^{n}$ is invariant under the flow defined by the equation, we show that the chain in question has a unique stationary measure on $S$. Moreover, it is proved that this measure is exponentially attracting in the variational norm. Once we have the uniqueness of stationary measure on the sphere $S$, using the invariance of $S$, the class of all stationary measures in $\mathbb{C}^{n}$ can be described.

The ergodicity of finite-dimensional stochastic systems is studied by many authors. Let us mention some earlier results in this direction. Uniqueness of stationary measure for non-degenerate diffusion processes is obtained by Hasminskii [10]. The case of the degenerate diffusions is considered by Arnold and Kliemann [6] and Veretennikov $[\mathbf{1 7}, \mathbf{1 8}]$. Various sufficient conditions for ergodicity of abstract Markov processes are obtained by Meyn and Tweedie in [14] and [13]. E and Mattingly $[\mathbf{9}]$ consider the finite-dimensional approximations of the 2D Navier-Stokes equations, and Romito [15] considers the approximations of the 3D Navier-Stokes equations. In both cases, the perturbation is an additive white noise and the main result is the exponential mixing in the variational norm.

We prove the ergodicity of system (1.1), (1.3) under some conditions over the matrices $\Lambda$ and $B$ and over the distribution of the random variable $\eta_{1}$. Roughly speaking, we assume that there is no proper vector space invariant under both $\Lambda$ and $B$, and that the support of the law of $\eta_{1}$ contains a ball of sufficiently high dimension. These conditions enable us to use a measure transformation theorem from $[\mathbf{1}]$ and some controllability results from $[\mathbf{7}]$ and [8]. Let us emphasize that the noise in system (1.1) is always one-dimensional, independently of the space dimension $n \geq 1$. Moreover, the distribution of the noise is degenerate and it is not supposed to have a Gaussian structure.

Our proof is based on a classical coupling argument combined with some controllability properties of the Schrödinger equation. It is divided into two steps. First, using the measure transformation theorem, we show that there is a ball $D \subset S$ and a constant $p \in(0,1)$ such that the variational distance at time $t=1$ between any two solutions issued from $D$ is less than $p$. Then we show that $D$ is accessible from any point of $S$. From the compactness of $S$ it follows that the first hitting time of $D$ admits an exponential estimate. Combination of the above properties with a suitable coupling construction gives the proof of the exponential mixing property.

Let us note that in the case of the diffusion process defined by the Stratonovich stochastic differential equation

$$
i \mathrm{~d} z=[\Lambda z+\varepsilon F(z)] \mathrm{d} t+B z \circ W(t),
$$


the uniqueness of stationary measure can be obtained as a consequence of [6]. Indeed, under the conditions imposed on $\Lambda$ and $B$, a direct verification shows that the Lie algebra generated by the drift and diffusion fields is full at the point $e_{1}$, where $e_{1}$ is the first eigenvector of the matrix $\Lambda$.

As an application of our result, we consider the Galerkin approximations of the Schrödinger equation with potential of random amplitude. We show that if the deterministic part of the potential is in a general position, then the property of exponential mixing holds for any finite-dimensional approximation. The technique developed in this paper can be applied to finite-dimensional approximations of other stochastic PDE's. In particular, using the controllability results of Agrachev and Sarychev $[\mathbf{2}]$ and Shirikyan [16], one can prove ergodicity of finite-dimensional approximations of $2 \mathrm{D}$ and $3 \mathrm{D}$ Navier-Stokes equations in the case of degenerate non-Gaussian forcing. In conclusion, let us note that even though our proof does not apply to the infinite-dimensional Schrödinger equation, many properties remain valid. In particular, an approximate controllability property holds for the Schrödinger equation, which enables one to show that almost any trajectory of randomly forced equation is unbounded in the Sobolev space of any order $s>0$. These questions will be addressed in a forthcoming paper.

Acknowledgments. The author is grateful to his advisor, Armen Shirikyan, for many helpful conversations and support.

\section{Notation}

In this paper, we use the following notation.

$S$ is the unit sphere in $\mathbb{C}^{n}$, i.e. $S=\left\{x \in \mathbb{C}^{n}:\|x\|_{\mathbb{C}^{n}}=1\right\} . S$ is regarded as a $(2 n-1)$-dimensional real-analytic manifold endowed with the standard Riemannian metric and the corresponding measure. The latter is denoted by $m$.

$T_{y} S$ is the tangent space to $S$ at the point $y \in S$, i.e. $T_{y} S=\left\{x \in \mathbb{C}^{n}: \operatorname{Re}\langle x, y\rangle=0\right\}$, where $\langle\cdot, \cdot\rangle$ stands for the scalar product in $\mathbb{C}^{n}$.

$C_{b}(S)$ is the space of real-valued continuous bounded functions on $S$ endowed with the norm $\|f\|_{\infty}:=\sup |f|$.

$\mathcal{B}(S)$ is the Borel $\sigma$-algebra of $S$.

$\mathcal{P}(S)$ is the set of probability measures on $(S, \mathcal{B}(S))$.

The set $\mathcal{P}(S)$ is endowed with the variational norm:

$$
\left\|\mu_{1}-\mu_{2}\right\|_{\text {var }}:=\sup _{\Gamma \in \mathcal{B}(S)}\left|\mu_{1}(\Gamma)-\mu_{2}(\Gamma)\right|, \quad \mu_{1}, \mu_{2} \in \mathcal{P}(S) .
$$

The distribution of a random variable $\xi$ is denoted by $\mathcal{D}(\xi)$.

The indicator function of a set $\Gamma$ is denoted by $I_{\Gamma}$.

For a metric space $E$, we denote by $B_{E}(a, r)$ the open ball of radius $r>0$ centered at $a \in E$. If $E=\mathbb{C}^{n}$, we simply write $B(a, r)$.

$\mathbb{I}$ denotes the set of irrational numbers.

\section{Main result}

2.1. Uniqueness and exponential mixing. Under the conditions described at the beginning of Section 1, for any $z_{0} \in \mathbb{C}^{n}$ problem (1.1), (1.2) has a unique solution almost surely belonging to the space $C\left([0, \infty), \mathbb{C}^{n}\right)$. Let $\mathcal{U}_{t}^{\varepsilon}: \mathbb{C}^{n} \rightarrow \mathbb{C}^{n}$ be 
the resolving operator of (1.1), (1.2). Note that

$$
\left\|\mathcal{U}_{t}^{\varepsilon}\left(z_{0}\right)\right\|_{\mathbb{C}^{n}}=\left\|z_{0}\right\|_{\mathbb{C}^{n}}, \quad t \geq 0 .
$$

Let $z_{0}$ be a $\mathbb{C}^{n}$-valued random variable independent of $\left\{\eta_{k}\right\}$. Denote by $\mathcal{F}_{k}$ the $\sigma$-algebra generated by $z_{0}, \eta_{0}, \ldots, \eta_{k-1}$. Then $\mathcal{U}_{k}^{\varepsilon}\left(z_{0}\right)$ is a homogeneous Markov chain with respect to $\mathcal{F}_{k}$. The corresponding transition function has the form $P_{k}^{\varepsilon}(z, \Gamma)=\mathbb{P}\left\{\mathcal{U}_{k}^{\varepsilon}(z) \in \Gamma\right\}, z \in \mathbb{C}^{n}, \Gamma \in \mathcal{B}\left(\mathbb{C}^{n}\right)$, and the Markov operator is defined as

$$
\mathfrak{P}_{k}^{\varepsilon *} \mu(\Gamma)=\int_{\mathbb{C}^{n}} P_{k}^{\varepsilon}(z, \Gamma) \mu(\mathrm{d} z),
$$

where $\mu \in \mathcal{P}\left(\mathbb{C}^{n}\right)$. Recall that a measure $\mu \in \mathcal{P}\left(\mathbb{C}^{n}\right)$ is called stationary for (1.1), (1.3) if $\mathfrak{P}_{1}^{\varepsilon *} \mu=\mu$.

It follows from (2.1) that the unit sphere $S$ is invariant under the flow defined by (1.1). The Bogolyubov-Krylov argument and the compactness of $S$ imply the existence of a stationary measure $\mu \in \mathcal{P}(S)$ for problem (1.1), (1.3).

To be able to show the uniqueness of stationary measure, we need the following conditions.

Condition 2.1. The random variables $\eta_{k}$ have the form

$$
\eta_{k}(t)=\sum_{j=1}^{\infty} b_{j} \xi_{j k} g_{j}(t), \quad t \in[0,1],
$$

where $\left\{g_{j}\right\}$ is an orthonormal basis in $L^{2}([0,1], \mathbb{R}), b_{j} \geq 0$ are constants with

$$
\sum_{j=1}^{\infty} b_{j}^{2}<\infty
$$

and $\xi_{j k}$ are independent real-valued random variables such that $\mathbb{E} \xi_{j k}^{2}=1$. Moreover, the distribution of $\xi_{j k}$ possesses a continuous density $\rho_{j}$ with respect to the Lebesgue measure and $\rho_{j}(r)>0$ for all $r \in \mathbb{R}$.

This condition is adapted to the hypotheses of a measure transformation theorem from [1]. In particular, under this condition, the image of measure $\mathcal{D}\left(\eta_{k}\right)$ under a large class of finite-dimensional transformations is absolutely continuous with respect to the Lebesgue measure.

Let $\left\{e_{j}\right\}_{j=1}^{n}$ be the set of normalized eigenvectors of $\Lambda$ with eigenvalues $\lambda_{1} \leq$ $\ldots \leq \lambda_{n}$.

Condition 2.2. The eigenvalues of $\Lambda$ are distinct and $\left\langle B e_{1}, e_{j}\right\rangle \neq 0, j=1, \ldots, n$.

Under this condition, some strong controllability properties hold for (1.1). In particular, the linearization of (1.1) is controllable, which, combined with the inverse function theorem, gives a local exact controllability property (see Section 3). Moreover, Condition 2.2 also allows us to use a stabilization result from [8]. Notice that, as in $[\mathbf{8}]$, all the results of the paper remain valid under the assumption that for some $i=1, \ldots, n$ we have $\left\langle B e_{i}, e_{j}\right\rangle \neq 0$ for all $j=1, \ldots, n$ and $\left|\lambda_{p}-\lambda_{i}\right| \neq\left|\lambda_{q}-\lambda_{i}\right|$ for all $p \neq q$. Clearly, in the case $i=1$, the last condition follows from the non-degeneracy of the spectrum of $\Lambda$.

The following theorem is our main result. 
THEOREM 2.3. Suppose that Conditions 2.1 and 2.2 are satisfied. Then there is an integer $N \geq 1$ and a constant $\varepsilon_{0}>0$ such that, if

$$
b_{j} \neq 0, \quad j=1, \ldots, N
$$

then problem (1.1), (1.3) has a unique stationary measure $\mu \in \mathcal{P}(S)$ for $|\varepsilon|<\varepsilon_{0}$. Moreover, $\mu$ is absolutely continuous with respect to the measure $m$, and for any initial measure $\nu \in \mathcal{P}(S)$, we have

$$
\left\|\mathfrak{P}_{k}^{\varepsilon *} \nu-\mu\right\|_{v a r} \leq C e^{-c k}, \quad k \geq 1
$$

where $C>0$ and $c>0$ are constants.

2.2. Proof of Theorem 2.3. The proof of Theorem 2.3 is derived from the two lemmas below. Their proofs are given in Section 4.

LEMMA 2.4. Under the conditions of Theorem 2.3, there are constants $\delta_{0}>0$, $\varepsilon_{0}>0$ and $p \in(0,1)$ and integers $N \geq 1$ and $l \geq 1$ such that, if (2.2) holds, then:

(i) For any $z, z^{\prime} \in S \cap B\left(e_{1}, \delta_{0}\right)$ and $|\varepsilon|<\varepsilon_{0}$, we have

$$
\left\|P_{1}^{\varepsilon}(z, \cdot)-P_{1}^{\varepsilon}\left(z^{\prime}, \cdot\right)\right\|_{\text {var }} \leq p .
$$

(ii) For any $z \in S$ and $|\varepsilon|<\varepsilon_{0}$, the measure $P_{l}^{\varepsilon}(z, \cdot)$ is absolutely continuous with respect to $m$.

For any $\delta>0$, let us introduce the stopping time

$$
\tau_{\delta, \varepsilon}=\min \left\{k \geq 0: \mathcal{U}_{k}^{\varepsilon}(z) \in B\left(e_{1}, \delta\right)\right\} .
$$

LEMma 2.5. Under the conditions of Theorem 2.3, for any $\delta>0$ there is a constant $\varepsilon_{\delta}>0$ and an integer $N \geq 1$ such that, if (2.2) holds, then

$$
\mathbb{E}_{z} e^{\alpha \tau_{\delta, \varepsilon}} \leq C \quad \text { for all } z \in S \text { and }|\varepsilon|<\varepsilon_{\delta},
$$

where $\alpha>0$ and $C>0$ are constants, and the subscript $z$ means that the expectation is taken for the chain issued from $z$.

Proof of Theorem 2.3. Step 1. Let $z_{0}, z_{0}^{\prime} \in S$. The idea of the proof is to construct two sequences $y_{k}$ and $y_{k}^{\prime}$ such that $\mathcal{D}\left(y_{k}\right)=P_{k}^{\varepsilon}\left(z_{0}, \cdot\right), \mathcal{D}\left(y_{k}^{\prime}\right)=P_{k}^{\varepsilon}\left(z_{0}^{\prime}, \cdot\right)$ and the following inequality holds

$$
\left\|\mathcal{D}\left(y_{k}\right)-\mathcal{D}\left(y_{k}^{\prime}\right)\right\|_{\text {var }} \leq C e^{-c k}, \quad k \geq 0 .
$$

A well-known argument shows that (2.5) implies (2.3) (e.g, see [11]).

Step 2. Let $z, z^{\prime} \in S$. If $z=z^{\prime}$, then define $V\left(z, z^{\prime}\right)=V^{\prime}\left(z, z^{\prime}\right)=\mathcal{U}_{1}^{\varepsilon}(z)$. Let $\delta_{0}>0$ and $\varepsilon_{0}>0$ be the constants in Lemma 2.4 and $|\varepsilon|<\varepsilon_{0}$. If $z \neq z^{\prime}$ and $z, z^{\prime} \in B\left(e_{1}, \delta_{0}\right)$, then let $V\left(z, z^{\prime}\right), V^{\prime}\left(z, z^{\prime}\right)$ be any maximal coupling for $\left(P_{1}^{\varepsilon}(z, \cdot), P_{1}^{\varepsilon}\left(z^{\prime}, \cdot\right)\right)$ (see $[\mathbf{1 2}]$, Section I.5). Otherwise, let $V\left(z, z^{\prime}\right)$ and $V^{\prime}\left(z, z^{\prime}\right)$ be the values at $t=1$ of the solutions of the following problems:

$$
\begin{array}{ll}
i \frac{\mathrm{d} y}{\mathrm{~d} t}=\Lambda y+\eta(t) B y+\varepsilon F(y), & i \frac{\mathrm{d} y^{\prime}}{\mathrm{d} t}=\Lambda y^{\prime}+\eta^{\prime}(t) B y^{\prime}+\varepsilon F\left(y^{\prime}\right), \\
y(0)=z, & y^{\prime}(0)=z^{\prime},
\end{array}
$$

where $\eta$ and $\eta^{\prime}$ are independent random variables with $\mathcal{D}(\eta)=\mathcal{D}\left(\eta^{\prime}\right)=\mathcal{D}\left(\eta_{1}\right)$. Let $V_{k}, V_{k}^{\prime}, k \geq 1$ be independent copies of the random variables $V$ and $V^{\prime}$ depending 
on the parameters $z$ and $z^{\prime}$. Let $y_{0}=z_{0}$ and $y_{0}^{\prime}=z_{0}^{\prime}$. Define $y_{k}$ and $y_{k}^{\prime}, k \geq 1$ by the relations

$$
\begin{aligned}
& y_{k}=V_{k}\left(y_{k-1}, y_{k-1}^{\prime}\right), \\
& y_{k}^{\prime}=V_{k}^{\prime}\left(y_{k-1}, y_{k-1}^{\prime}\right) .
\end{aligned}
$$

Clearly, $\left(y_{k}, y_{k}^{\prime}\right)$ is a Markov chain. It is easy to see that $\mathcal{D}\left(y_{k}\right)=P_{k}^{\varepsilon}\left(z_{0}, \cdot\right)$ and $\mathcal{D}\left(y_{k}^{\prime}\right)=P_{k}^{\varepsilon}\left(z_{0}^{\prime}, \cdot\right)$. Define

$$
T=\min \left\{k \geq 0: y_{k}, y_{k}^{\prime} \in B\left(e_{1}, \delta_{0}\right)\right\} .
$$

Using the same arguments as in the proof of (2.4), one can show that, if $\varepsilon_{0}>0$ is sufficiently small, then

$$
\mathbb{E} e^{\alpha T} \leq C \text { for }|\varepsilon|<\varepsilon_{0},
$$

where $\alpha>0$ and $C>0$ are some constants not depending on $\varepsilon$ (see Remark 4.1).

Step 3. Suppose that there is a random integer $\ell$ such that

$$
\begin{aligned}
& y_{k}=y_{k}^{\prime} \text { for all } k \geq \ell, \\
& \mathbb{E} e^{\gamma \ell} \leq C .
\end{aligned}
$$

Then (2.8) and (2.9) imply (2.5). Indeed, for any $f \in C_{b}(S),\|f\|_{\infty} \leq 1$, we have

$$
\begin{aligned}
\left|\mathbb{E}\left(f\left(y_{k}\right)-f\left(y_{k}^{\prime}\right)\right)\right| & \leq \mathbb{E}\left|\left(f\left(y_{k}\right)-f\left(y_{k}^{\prime}\right)\right)\right| \\
& \leq \mathbb{E}\left|\left(f\left(y_{k}\right)-f\left(y_{k}^{\prime}\right)\right) I_{\{k \geq \ell\}}\right|+\mathbb{E}\left|\left(f\left(y_{k}\right)-f\left(y_{k}^{\prime}\right)\right) I_{\{k<\ell\}}\right| \\
& \leq 2 \mathbb{P}\{k<\ell\} \leq 2 C e^{-\gamma k},
\end{aligned}
$$

which proves (2.5).

Step 4. Let us introduce the stopping times $T(0)=0, T(1)=T$ and

$$
T(n)=\min \left\{k>T(n-1): y_{k}, y_{k}^{\prime} \in B\left(e_{1}, \delta_{0}\right)\right\}, \quad n \geq 2 \text {. }
$$

Using the strong Markov property and (2.7), we see that

$$
\mathbb{E} e^{\alpha T(n)}=\mathbb{E} e^{\alpha T(n-1)} \mathbb{E}_{Y(n)} e^{\alpha T} \leq C \mathbb{E} e^{\alpha T(n-1)},
$$

where $Y(n)=\left(y_{T(n-1)}, y_{T(n-1)}^{\prime}\right)$. Thus

$$
\mathbb{E} e^{\alpha T(n)} \leq C^{n}
$$

Define

$$
\ell=\min \left\{k \geq 0: y_{n}=y_{n}^{\prime} \text { for all } n \geq k\right\},
$$

where $\min \{\emptyset\}=\infty$. Let us show that

$$
\mathbb{P}\{\ell>T(n+1)\} \leq p^{n},
$$

where $p \in(0,1)$ is the constant in Lemma 2.4. Indeed, it follows from Lemma 2.4 and the construction of $y_{k}$ and $y_{k}^{\prime}$ that

$$
\begin{aligned}
\mathbb{P}\left\{y_{T(n)+1} \neq y_{T(n)+1}^{\prime}\right\} & =\mathbb{P}\left\{y_{T(n)+1} \neq y_{T(n)+1}^{\prime} \mid y_{T(n)} \neq y_{T(n)}^{\prime}\right\} \mathbb{P}\left\{y_{T(n)} \neq y_{T(n)}^{\prime}\right\} \\
& \leq p \mathbb{P}\left\{y_{T(n)} \neq y_{T(n)}^{\prime}\right\} \leq p \mathbb{P}\left\{y_{T(n-1)+1} \neq y_{T(n-1)+1}^{\prime}\right\} .
\end{aligned}
$$

Iteration of this inequality gives

$$
\mathbb{P}\left\{y_{T(n)+1} \neq y_{T(n)+1}^{\prime}\right\} \leq p^{n} .
$$

On the other hand, the definition of $\ell$ implies that

$$
\mathbb{P}\{\ell>T(n+1)\} \leq \mathbb{P}\left\{y_{T(n)+1} \neq y_{T(n)+1}^{\prime}\right\},
$$


which proves (2.11). Thus, by the Borel-Cantelli lemma, we have $\mathbb{P}\{\ell<\infty\}=1$.

Let $r>0$ be so large that $C^{\frac{1}{r}} p^{1-\frac{1}{r}}<1$, and let $c$ be so small that $r c<\alpha$. Using the Cauchy-Schwarz inequality and (2.11), we obtain

$$
\begin{aligned}
\mathbb{E} e^{c \ell} & \leq 1+\sum_{n=0}^{\infty} \mathbb{E}\left(I_{\{T(n)<\ell \leq T(n+1)\}} e^{c \ell}\right) \\
& \leq 1+\sum_{n=0}^{\infty} \mathbb{E}\left(I_{\{T(n)<\ell \leq T(n+1)\}} e^{c T(n+1)}\right) \\
& \leq 1+\sum_{n=0}^{\infty}\left(\mathbb{E} e^{r c T(n+1)}\right)^{\frac{1}{r}} \mathbb{P}\{\ell>T(n)\}^{1-\frac{1}{r}} \\
& \leq 1+C^{\frac{1}{r}} p^{\frac{1}{r}-1} \sum_{n=0}^{\infty}\left(C^{\frac{1}{r}} p^{1-\frac{1}{r}}\right)^{n}<\infty .
\end{aligned}
$$

This completes the proof of (2.3).

Step 5. To show that the stationary measure $\mu$ is absolutely continuous with respect to the Riemannian volume $m$ on $S$, take any $\Gamma \in \mathcal{B}(S)$ such that $m(\Gamma)=0$ and let $l \geq 1$ be the integer in Lemma 2.4. Then

$$
\mu(\Gamma)=\int_{S} P_{l}^{\varepsilon}(z, \Gamma) \mu(\mathrm{d} z)=0,
$$

as $P_{l}^{\varepsilon}(z, \cdot)$ is absolutely continuous with respect to $m$ for any $z \in S$.

2.3. Stationary measures in $\mathbb{C}^{n}$. Any measure $\nu \in \mathcal{P}\left(\mathbb{C}^{n}\right)$ can be written in the form

$$
\nu=\alpha \delta_{0}+(1-\alpha) \bar{\nu}
$$

where $\alpha \in[0,1], \delta_{0}$ is the Dirac measure concentrated at zero and $\bar{\nu} \in \mathcal{P}\left(\mathbb{C}^{n} \backslash\{0\}\right)$. Indeed, it suffices to take $\alpha=\nu(\{0\})$ and $\bar{\nu}(\cdot)=\frac{1}{1-\alpha} \nu\left(\cdot \cap \mathbb{C}^{n} \backslash\{0\}\right)$, if $\alpha<1$. On the other hand, for any measure $\bar{\nu} \in \mathcal{P}\left(\mathbb{C}^{n} \backslash\{0\}\right)$ there is a measure $\gamma \in \mathcal{P}\left(\mathbb{R}_{+}^{*}\right)$ and a random measure $\mu_{r} \in \mathcal{P}(S), r \in \mathbb{R}_{+}^{*}$ (i.e. for any $\Gamma \in \mathcal{B}(S)$ the function $r \rightarrow \mu_{r}(\Gamma)$ is measurable) such that for any bounded measurable function $f: \mathbb{C}^{n} \backslash\{0\} \rightarrow \mathbb{R}$ we have

$$
\int_{\mathbb{C}^{n} \backslash\{0\}} f(v) \bar{\nu}(\mathrm{d} v)=\int_{\mathbb{R}_{+}^{*}} \int_{S} f(r u) \mu_{r}(\mathrm{~d} u) \gamma(\mathrm{d} r)
$$

(e.g., see [5]). In this case, we write

$$
\bar{\nu}(\mathrm{d} r, \mathrm{~d} u)=\mu_{r}(\mathrm{~d} u) \gamma(\mathrm{d} r) .
$$

Let $\mu \in \mathcal{P}(S)$ be the stationary measure in Theorem 2.3 for $\varepsilon=0$, i.e. corresponding to the linear equation.

THEOREM 2.6. Under the conditions of Theorem 2.3, there is an integer $N \geq 1$ such that, if (2.2) holds, then a measure $\nu \in \mathcal{P}\left(\mathbb{C}^{n}\right)$ is stationary for problem (1.1), (1.3) with $\varepsilon=0$ if and only if it can be represented in the form (2.12), (2.14) in a way that $\mu_{r}=\mu$ for $\gamma$-almost all $r \in \mathbb{R}_{+}^{*}$. Moreover, for any initial measure $\nu^{\prime} \in \mathcal{P}\left(\mathbb{C}^{n}\right)$ of the form

$$
\begin{aligned}
\nu^{\prime} & =\alpha \delta_{0}+(1-\alpha) \bar{\nu}^{\prime}, \\
\bar{\nu}^{\prime}(\mathrm{d} r, \mathrm{~d} u) & =\mu_{r}^{\prime}(\mathrm{d} u) \gamma(\mathrm{d} r),
\end{aligned}
$$


we have

$$
\left\|\mathfrak{P}_{k}^{0 *} \nu^{\prime}-\nu\right\|_{v a r} \leq C e^{-c k}, \quad k \geq 1,
$$

where $C>0$ and $c>0$ are constants.

Proof. Without loss of generality, we can assume that $\alpha=0$, i.e. $\nu=\bar{\nu} \in$ $\mathcal{P}\left(\mathbb{C}^{n} \backslash\{0\}\right)$. Suppose that $\nu$ is a stationary measure. Let us show that $\mu_{r}$ is stationary for $\gamma$-almost all $r \in \mathbb{R}_{+}^{*}$. Take any bounded measurable functions $f$ : $S \rightarrow \mathbb{R}$ and $g: \mathbb{R}_{+}^{*} \rightarrow \mathbb{R}$. By $(2.13)$, we have

$$
\begin{aligned}
\int_{\mathbb{C}^{n} \backslash\{0\}}(f g)(v) \nu(\mathrm{d} v) & =\int_{\mathbb{R}_{+}^{*}} g(r) \int_{S} f(u) \mu_{r}(\mathrm{~d} u) \gamma(\mathrm{d} r) \\
& =\mathbb{E} \int_{\mathbb{C}^{n} \backslash\{0\}}(f g)\left(\mathcal{U}_{1}^{0}(v)\right) \nu(\mathrm{d} v) \\
& =\mathbb{E} \int_{\mathbb{C}^{n} \backslash\{0\}} f\left(\frac{\mathcal{U}_{1}^{0}(v)}{\left\|\mathcal{U}_{1}^{0}(v)\right\|}\right) g\left(\left\|\mathcal{U}_{1}^{0}(v)\right\|\right) \nu(\mathrm{d} v) \\
& =\int_{\mathbb{R}_{+}^{*}} g(r)\left[\mathbb{E} \int_{S} f\left(\mathcal{U}_{1}^{0}(u)\right) \mu_{r}(\mathrm{~d} u)\right] \gamma(\mathrm{d} r),
\end{aligned}
$$

where we used the fact that $\mathcal{U}_{t}^{0}(v)$ is a solution of a linear equation. As (2.16) holds for any bounded measurable functions $g$ and $f$ and the Borel $\sigma$-algebras on $S$ and $\mathbb{R}_{+}^{*}$ are countably generated, we see that $\mu_{r}$ is stationary for $\gamma$-almost all $r \in \mathbb{R}_{+}^{*}$. By the uniqueness of stationary measure, we get $\mu_{r}=\mu$ for $\gamma$-almost all $r \in \mathbb{R}_{+}^{*}$.

On the other hand, if $\mu_{r}=\mu$ for $\gamma$-almost all $r \in \mathbb{R}_{+}^{*}$ and $\nu$ is defined by (2.14), then by a similar argument, one can prove that $\nu$ is a stationary measure.

To prove the second assertion, let us take any bounded measurable functions $f: S \rightarrow \mathbb{R}$ and $g: \mathbb{R}_{+}^{*} \rightarrow \mathbb{R}$ such that $\sup |f| \leq 1$ and $\sup |g| \leq 1$. Then

$$
\begin{aligned}
& \mathbb{E}\left|\int_{\mathbb{C}^{n} \backslash\{0\}}(f g)\left(\mathcal{U}_{k}^{0}(v)\right) \nu^{\prime}(\mathrm{d} v)-\int_{\mathbb{C}^{n} \backslash\{0\}}(f g)(v) \nu(\mathrm{d} v)\right| \\
& \left.\leq \mathbb{E} \int_{\mathbb{R}_{+}^{*}}|g(r)| \mid \int_{S} f\left(\mathcal{U}_{k}^{0}(u)\right)\right) \mu_{r}(\mathrm{~d} u)-\int_{S} f(u) \mu(\mathrm{d} u) \mid \gamma(\mathrm{d} r) \\
& \leq C e^{-c k} .
\end{aligned}
$$

The general case is obtained by the monotone class theorem.

REMARK 2.7. Denote by $S_{r}$ the sphere of radius $r>0$ in $\mathbb{C}^{n}$. Let $N(r) \geq 1$ and $\varepsilon_{0}(r)>0$ be the constants in Theorem 2.3 applied for the sphere $S_{r}$, and let $\mu_{r} \in \mathcal{P}\left(S_{r}\right)$ be the stationary measure for $\varepsilon \in\left(0, \varepsilon_{0}(r)\right)$. In this case, the projections of measures $\mu_{r}$ to $S$ depend on $r>0$. On the other hand, $N(r) \rightarrow \infty$ and $\varepsilon_{0}(r) \rightarrow 0$ as $r \rightarrow \infty$. Taking into account these facts, one can reformulate Theorem 2.6 for measures $\nu \in \mathcal{P}(B(0, r))$.

\section{Controllability results}

Let us consider the control system

$$
\begin{aligned}
i \frac{\mathrm{d} z}{\mathrm{~d} t} & =\Lambda z+u(t) B z, \\
z(0) & =z_{0},
\end{aligned}
$$


where the state is $z$ and the control is $u$. Let $\mathcal{R}_{t}(\cdot, u): S \rightarrow S$ be the resolving operator of $(3.1),(3.2)$. Recall that $\Lambda$ and $B$ satisfy Condition 2.2.

THEOREM 3.1. System (3.1), (3.2) is globally exactly controllable, i.e. for any $z_{1}, z_{2} \in S$ there is a time $T \geq 0$ and a control $u \in L^{2}([0, T], \mathbb{R})$ such that $\mathcal{R}_{T}\left(z_{1}, u\right)=z_{2}$.

The proof of this theorem can be derived from Theorem 4 in [4], where a necessary and sufficient condition for the controllability of (3.1) is stated. One can verify that the condition given in [4] is weaker than Condition 2.2. Here we give another proof of Theorem 3.1. This proof provides some additional information on the control (see Remark 3.4), which is important for the application in the proof of Theorem 2.3.

The proof of Theorem 3.1 is based on several ideas of [7] and [8]. It is derived from two lemmas below, which are of independent interest. We postponed the proof of Theorem 3.1 to the end of this section.

Let us introduce the following $(2 n-1)$-dimensional subspace of $L^{2}([0,1], \mathbb{R})$ :

$$
E_{n}=\left\{v \in L^{2}([0,1]): v(t)=\sum_{k=-(n-1)}^{n-1} d_{k} e^{i \mu_{k} t}, d_{k} \in \mathbb{C}, d_{-k}=\bar{d}_{k}, t \in[0,1]\right\}
$$

where $\mu_{k}=\lambda_{k+1}-\lambda_{1}$ and $\mu_{-k}=-\mu_{k}$.

LEMMA 3.2. For any $\nu>0$ there is a constant $\delta>0$ such that for any $z_{i} \in$ $S \cap B\left(e_{1}, \delta\right)$ and $z_{f} \in S \cap B\left(e_{1} e^{-i \lambda_{1}}, \delta\right)$ there is a control $u \in B_{E_{n}}(0, \nu)$ satisfying $\mathcal{R}_{1}\left(z_{i}, u\right)=z_{f}$.

Proof. We follow the ideas of [7], where the local exact controllability of an infinite-dimensional Schrödinger equation is proved using the Nash-Moser implicit function theorem. In our situation, the controllability is derived from the inverse function theorem.

For any $z \in S$ and $u \in E_{n}$, define $\Phi(z, u)=\left(z, \mathcal{R}_{1}(z, u)\right)$. Note that $\Phi\left(e_{1}, 0\right)=$ $\left(e_{1}, e_{1} e^{-i \lambda_{1}}\right)$. We are going to show that the conditions of inverse mapping theorem are satisfied in a neighborhood of the point $\left(e_{1}, 0\right) \in S \times E_{n}$. Clearly, $\Phi$ is continuously differentiable. Let us show that mapping $D \Phi\left(e_{1}, 0\right): T_{e_{1}} S \times E_{n} \rightarrow$ $T_{e_{1}} S \times T_{e_{1} e^{-i \lambda_{1}}} S$ is an isomorphism. Consider the linearization of (3.1), (3.2) around $\left(e_{1} e^{-i \lambda_{1} t}, 0\right)$ :

$$
\begin{aligned}
i \frac{\mathrm{d} y}{\mathrm{~d} t} & =\Lambda y+w(t) B e_{1} e^{-i \lambda_{1} t}, \\
y(0) & =y_{0},
\end{aligned}
$$

where $w \in E_{n}$ and $y_{0} \in T_{e_{1}} S$. Denote by $y_{t}=y_{t}\left(y_{0}, w\right)$ the solution of problem (3.3), (3.4). One can verify that $D \Phi\left(e_{1}, 0\right)\left(y_{0}, w\right)=\left(y_{0}, y_{1}\right)$. Note that $(3.3),(3.4)$ is equivalent to

$$
y_{t}=e^{-i \Lambda t} y_{0}-i \int_{0}^{t} e^{-i \Lambda(t-s)} w(s) B e_{1} e^{-i \lambda_{1} s} \mathrm{~d} s .
$$

Let $B_{i j}=\left\langle B e_{i}, e_{j}\right\rangle, i, j=1, \ldots, n$. Taking the scalar product of (3.5) with $e_{k} e^{-i \lambda_{k}}$, we obtain for $t=1$

$$
\left\langle y_{1}, e_{k} e^{-i \lambda_{k}}\right\rangle=\left\langle y_{0}, e_{k}\right\rangle-i B_{1 k} \int_{0}^{1} e^{i \mu_{k-1} s} w(s) \mathrm{d} s .
$$


Clearly $y_{1}\left(y_{0}, w\right) \in T_{e_{1} e^{-i \lambda_{1}}} S$, if $y_{0} \in T_{e_{1}} S$. Let $y_{1}^{\prime} \in T_{e_{1} e^{-i \lambda_{1}}} S$. By Condition 2.2, we have $B_{1 k} \neq 0$. Hence, the equality $y_{1}\left(y_{0}, w\right)=y_{1}^{\prime}$ is equivalent to

$$
c_{k}:=\frac{\left\langle y_{1}^{\prime}, e_{k} e^{-i \lambda_{k}}\right\rangle-\left\langle y_{0}, e_{k}\right\rangle}{-i B_{1 k}}=\int_{0}^{1} e^{i \mu_{k-1} s} w(s) \mathrm{d} s, \quad k=1, \ldots, n .
$$

Since $\left(y_{0}, y_{1}^{\prime}\right) \in T_{e_{1}} S \times T_{e_{1} e^{-i \lambda_{1}}} S$ and $B_{11} \in \mathbb{R}$, we have $c_{1} \in \mathbb{R}$. As the functions $\left\{e^{i \mu_{k} s}\right\}_{k=-(n-1)}^{n-1}$ are linearly independent, there is a unique solution $w \in$ $\operatorname{span}\left\{e^{i \mu_{k} s}\right\}_{k=-(n-1)}^{n-1}$ of the problem

$$
c_{k}=\int_{0}^{1} e^{i \mu_{k-1} s} w(s) \mathrm{d} s, \quad \bar{c}_{k}=\int_{0}^{1} e^{-i \mu_{k-1} s} w(s) \mathrm{d} s, \quad k=1, \ldots, n .
$$

Then $w=\bar{w}$, as $\bar{w}$ is a solution of the same problem. Thus $w \in E_{n}$. This shows the surjectivity of $D \Phi\left(e_{1}, 0\right)$. Finally, applying the inverse mapping theorem, we conclude that $\Phi$ is a $C^{1}$ diffeomorphism in the neighborhood of $\left(e_{1}, 0\right)$.

For any $u \in L^{2}([0, l]), l \in \mathbb{N}$, define $u_{j} \in L^{2}([0,1])$ as follows:

$$
u_{j}=\left.u(j+\cdot)\right|_{[0,1]}, \quad j=0, \ldots, l-1 .
$$

Lemma 3.3. For any $\nu>0, \delta>0$ and $s \in \mathbb{R}$ the following assertions hold.

(i) For any $z_{0} \in S$ there is a time $l \in \mathbb{N}$ and a control $u \in L^{2}([0, l], \mathbb{R})$ such that $\mathcal{R}_{l}\left(z_{0}, u\right) \in S \cap B\left(e_{1} e^{i s}, \delta\right)$.

(ii) There is an integer $N \geq 1$ such that for any $z_{0} \in S$ the control $u$ in (i) can be chosen in a way that

$$
u_{j} \in \operatorname{span}\left\{g_{1}, \ldots, g_{N}\right\} \quad \text { and }\left\|u_{j}\right\|_{L^{2}([0,1])} \leq \nu, j=0, \ldots, l-1
$$

Proof. Step 1. To prove (i), note that, without loss of generality, we can assume that the first eigenvalue of $\Lambda$ is of the form $\lambda_{1}=2 \pi \alpha$, where $\alpha \in \mathbb{I}$.

Indeed, for any $\gamma \in \mathbb{R}$, define the matrix $\Lambda_{\gamma}:=\Lambda+\gamma B$. Clearly, $\Lambda_{\gamma}$ is an Hermitian matrix and Condition 2.2 is satisfied, if $|\gamma|$ is sufficiently small. Let $\left\{\lambda_{k, \gamma}\right\}$ and $\left\{e_{k, \gamma}\right\}$ be the sets of eigenvalues and normalized eigenvectors of $\Lambda_{\gamma}$. Clearly, the resolving operator of problem (3.1), (3.2) with $\Lambda$ replaced by $\Lambda_{\gamma}$ is $\mathcal{R} .(\cdot, \cdot+\gamma)$. First let us show that it is possible to choose a sequence $\gamma_{n} \rightarrow 0$ such that $\lambda_{1, \gamma_{n}}=2 \pi \alpha_{n}$, where $\alpha_{n} \in \mathbb{I}$. Indeed, suppose that for some $\eta>0$ we have $\lambda_{1, \gamma}=\lambda_{1}$ for any $\gamma \in(-\eta, \eta)$. Then $\operatorname{det}\left(\Lambda_{\gamma}-\lambda_{1} I\right)=0$ for any $\gamma \in(-\eta, \eta)$, where $I$ is the $n \times n$ identity matrix. But $\operatorname{det}\left(\Lambda_{\gamma}-\lambda_{1} I\right)$ is a polynomial in $\gamma$, and the coefficient of the first order term is $\left\langle B e_{1}, e_{1}\right\rangle\left(\lambda_{2}-\lambda_{1}\right) \cdot \ldots \cdot\left(\lambda_{n}-\lambda_{1}\right)$, which is not zero by Condition 2.2. This contradiction shows that above-mentioned choice of the sequence $\gamma_{n}$ is possible.

If (i) holds for problem (3.1), (3.2) with $\Lambda$ replaced by $\Lambda_{\gamma_{n}}$, then there are sequences $l_{n} \in \mathbb{N}$ and $u_{n} \in L^{2}\left(\left[0, l_{n}\right], \mathbb{R}\right)$ such that $\mathcal{R}_{l_{n}}\left(z_{0}, \gamma_{n}+u_{n}\right) \in S \cap B\left(e_{1, \gamma_{n}} e^{i s}, \frac{\delta}{2}\right)$. If $n$ is sufficiently large, we have $e_{1, \gamma_{n}} \in B\left(e_{1}, \frac{\delta}{2}\right)$, thus $\gamma_{n}+u_{n}$ is the desired control.

Thus, we can suppose that $\lambda_{1}=2 \pi \alpha$, where $\alpha \in \mathbb{I}$. It follows that the set $\left\{e^{-i \lambda_{1} k}: k \in \mathbb{N}\right\}$ is dense in the circle $\{z \in \mathbb{C}:|z|=1\}$.

Step 2. Here we prove that (i) holds, if $\lambda_{1}=2 \pi \alpha, \alpha \in \mathbb{I}$. Define $\mathcal{C}=\left\{e_{1} e^{i t}\right.$ : $t \in \mathbb{R}\}$. It suffices to show that

(a) For any $z_{0} \in S$ there is a time $l \in \mathbb{N}$ and a control $u \in L^{2}([0, l], \mathbb{R})$ such that $\mathcal{R}_{l}\left(z_{0}, u\right) \in \mathcal{C}_{\delta}:=\{y \in S: \operatorname{dist}(y, \mathcal{C}) \leq \delta\}$. 
(b) For any $z_{0} \in \mathcal{C}_{\delta}$ there is a time $k \in \mathbb{N}$ and a control $v \in L^{2}([0, k], \mathbb{R})$ such that $\mathcal{R}_{k}\left(z_{0}, v\right) \in S \cap B\left(e_{1} e^{i s}, \delta\right)$.

To prove (a), following the ideas of [8], we introduce the feedback design

$$
u(z)=c \operatorname{Im}\left(\left\langle B z, e_{1}\right\rangle\left\langle e_{1}, z\right\rangle\right),
$$

where $c>0$ is a small constant. Let us consider the problem

$$
\begin{aligned}
& i \frac{\mathrm{d} z}{\mathrm{~d} t}=\Lambda z+u(z) B z, \\
& z(0)=z_{0} .
\end{aligned}
$$

As in $[8]$, one can show that for any $z_{0} \in S$ such that $\left\langle z_{0}, e_{1}\right\rangle \neq 0$, we have

$$
\lim _{t \rightarrow \infty} \operatorname{dist}\left(\mathcal{R}_{t}\left(z_{0}, u(z(t))\right), \mathcal{C}\right)=0 .
$$

The proof of our case is easier, as the linearization of (3.1), (3.2) around the trajectory $\left(e_{1}, u \equiv 0\right)$ is controllable. Now (a) follows from the fact that the set $\left\{z_{0} \in S:\left\langle z_{0}, e_{1}\right\rangle \neq 0\right\}$ is dense in $S$ and the distance between two solutions of (3.1) corresponding to the same control is constant.

The proof of (b) follows from the fact that $\lambda_{1}=2 \pi \alpha, \alpha \in \mathbb{I}$. Indeed, consider the solution of $(3.1),(3.2)$ with control $u \equiv 0$, that is $e^{-i \Lambda t} z_{0}$. As $z_{0} \in \mathcal{C}_{\delta}$, there is a $\tau \in \mathbb{R}$ such that $\left\|z_{0}-e_{1} e^{i \tau}\right\|_{\mathbb{C}^{n}}<\delta$. Clearly, the set $\left\{e_{1} e^{-i \lambda_{1} k+i \tau}: k \geq 1\right\}$ is dense in $\mathcal{C}$ and

$$
\left\|e^{-i \Lambda k} z_{0}-e_{1} e^{-i \lambda_{1} k+i \tau}\right\|_{\mathbb{C}^{n}}=\left\|z_{0}-e_{1} e^{i \tau}\right\|_{\mathbb{C}^{n}}<\delta .
$$

Thus $e^{-i \Lambda k} z_{0} \in B\left(e_{1} e^{i s}, \delta\right)$ for some $k \in \mathbb{N}$. This completes the proof of (i).

Step 3. To prove (ii), note that any $z_{0} \in S$ has a neighborhood whose points are controlled to $S \cap B\left(e_{1} e^{i s}, \delta\right)$ with controls from $\operatorname{span}\left\{g_{1}, \ldots, g_{N}\right\}$ for some $N:=$ $N\left(z_{0}\right)$. As $S$ is compact, we can find a universal constant $N$. The second part of (ii) follows directly from the construction (note that we can choose the constant $c$ in (3.9) arbitrarily small).

Proof of TheOrem 3.1. Step 1. Take any $z_{1}, z_{2} \in S$. Thank to Lemma 3.2 , it suffices to find controls $u_{j} \in L^{2}\left(\left[0, T_{j}\right], \mathbb{R}\right), T_{j}>0, j=1,2$ and a point $y \in S \cap B\left(e_{1} e^{-i \lambda_{1}}, \delta\right)$ such that $\mathcal{R}_{T_{1}}\left(z_{1}, u_{1}\right) \in S \cap B\left(e_{1}, \delta\right)$ and $\mathcal{R}_{T_{2}}\left(y, u_{2}\right)=z_{2}$.

Step 2. Clearly, we can take as $u_{1}$ the control provided by Lemma 3.3 for $z_{0}=z_{1}$ and $s=0$.

To construct the control $u_{2}$ and the point $y$, first note that, if $\mathcal{R}_{T}(\bar{z}, u)=\bar{y}$, then $\mathcal{R}_{T}\left(y, u^{\prime}\right)=z$, where $u^{\prime}(\cdot)=u(T-\cdot)$. Now let $u \in L^{2}\left(\left[0, T_{2}\right], \mathbb{R}\right)$ be the control provided by Lemma 3.3 for $z_{0}=\bar{z}_{2}$ and $s=\lambda_{1}$. It remains to take $u_{2}(\cdot)=u\left(T_{2}-\cdot\right)$ and $y=\overline{\mathcal{R}_{T}\left(\bar{z}_{2}, u\right)}$. Clearly, $y \in S \cap B\left(e_{1} e^{-i \lambda_{1}}, \delta\right)$ and $\mathcal{R}_{T_{2}}\left(y, u_{2}\right)=z_{2}$.

REMARK 3.4. It follows from the proof of Theorem 3.1 that for any $\nu>0$ there is an integer $N \geq 1$ such that the control $u$ and the time $T$ can be chosen in a way that $T \in \mathbb{N}$ and (3.8) is verified.

Now let us consider the system

$$
\begin{aligned}
i \frac{\mathrm{d} z}{\mathrm{~d} t} & =\Lambda z+u(t) B z+\varepsilon F(z), \\
z(0) & =z_{0} .
\end{aligned}
$$


Let $\mathcal{R}_{t}^{\varepsilon}(\cdot, u): S \rightarrow S$ be the resolving operator of (3.10), (3.11). Clearly, $\mathcal{R}_{t}^{0} \equiv \mathcal{R}_{t}$.

TheOREM 3.5. For any $\nu>0$ and $\delta>0$ there is a constant $\varepsilon_{0}=\varepsilon_{0}(\nu, \delta)>0$ and integers $N \geq 1$ and $k \geq 1$ such that following assertions hold.

(i) For any $z \in S$ there is a control $u \in L^{2}([0, k], \mathbb{R})$ such that $\mathcal{R}_{k}^{\varepsilon}(z, u) \in$ $B\left(e_{1}, \delta\right)$ for all $|\varepsilon|<\varepsilon_{0}$.

(ii) For any $z \in S$ the control $u$ in (i) can be chosen such that (3.8) is verified.

Notice that the time $k \geq 1$ is the same for all $z \in S$.

Proof. Step 1. First we prove (i) for $\varepsilon=0$. Let us choose points $x_{j} \in S$, $j=1, \ldots, q$ such that $S \subset \cup_{j=1}^{q} B\left(x_{j}, \frac{\delta}{2}\right)$ and $\left\langle x_{j}, e_{1}\right\rangle \neq 0$. Using the arguments in Step 1 of the proof of Lemma 3.3, one can choose small constants $\gamma_{j}, j=1, \ldots, q$ such that $\lambda_{1, \gamma_{j}}=2 \pi \alpha_{j}$ and $\left\{1, \alpha_{1}, \ldots, \alpha_{q}\right\}$ are rationally independent, where $\lambda_{1, \gamma}$ stands for the first eigenvalue of the matrix $\Lambda_{\gamma}=\Lambda+\gamma B$. Then using the feedback (3.9), we construct a time $l \geq 1$ and controls $v_{j} \in L^{2}([0, l], \mathbb{R})$ such that

$$
\operatorname{dist}\left(\mathcal{R}_{l}\left(x_{j}, \gamma_{j}+v_{j}\right), \mathcal{C}_{\gamma_{j}}\right) \leq \frac{\delta}{2},
$$

where $\mathcal{C}_{\gamma}=\left\{e_{1, \gamma} e^{i t}: t \in \mathbb{R}\right\}$ and $e_{1, \gamma}$ is the eigenvector corresponding to $\lambda_{1, \gamma}$. Let $\left|\gamma_{j}\right|$ be so small that $\left\|e_{1}-e_{1, \gamma_{j}}\right\| \leq \frac{\delta}{4}$. From (3.12) and the fact that $\left\{1, \alpha_{1}, \ldots, \alpha_{q}\right\}$ are rationally independent it follows the existence of an integer $l^{\prime} \geq 1$ such that $e^{-i \Lambda_{\gamma_{j}} l^{\prime}} \mathcal{R}_{k}\left(z_{j}, \gamma_{j}+v_{j}\right) \in B\left(e_{1, \gamma_{j}}, \frac{\delta}{4}\right)$. Thus we have constructed controls $u_{j} \in$ $L^{2}([0, k], \mathbb{R}), k:=l+l^{\prime}$ such that $\mathcal{R}_{k}\left(x_{j}, u_{j}\right) \in B\left(e_{1}, \frac{\delta}{2}\right)$. As in the case $\varepsilon=0$ the distance between two solutions is constant and $S \subset \cup_{j=1}^{q} B\left(x_{j}, \frac{\delta}{2}\right)$, for any $z \in S$ there is an integer $j \in[1, q]$ such that $\mathcal{R}_{k}\left(z, u_{j}\right) \in B\left(e_{1}, \delta\right)$. Clearly, as in Step 3 in the proof of Lemma 3.3, we can suppose that the controls $u_{j}$ satisfy assertion (ii).

Step 2. Let us prove the lemma in the general case. Let $z_{0} \in S$ and let $u \in L^{2}([0, k], \mathbb{R})$ be the control constructed in Step 1. It is easy to see that

$$
\lim _{(z, \varepsilon) \rightarrow\left(z_{0}, 0\right)} \mathcal{R}_{k}^{\varepsilon}(z, u)=\mathcal{R}_{k}\left(z_{0}, u\right) .
$$

Thus there is a constant $\varepsilon\left(z_{0}\right)>0$ such that $\mathcal{R}_{k}^{\varepsilon}(z, u) \in B\left(e_{1}, \delta\right)$ for all $|\varepsilon|<\varepsilon_{0}\left(z_{0}\right)$ and $z \in S \cap B\left(z_{0}, \varepsilon_{0}\left(z_{0}\right)\right)$. From the compactness of $S$ it follows that there is a uniform constant $\varepsilon_{0}>0$ such that assertions (i) and (ii) of Theorem 3.5 are satisfied.

\section{Proof of Lemmas 2.4 and 2.5}

Proof of Lemma 2.4. Step 1. To prove assertion (i), let us consider the mapping

$$
\begin{aligned}
\mathcal{R}_{1}(\cdot, \cdot):\left(-\varepsilon_{0}, \varepsilon_{0}\right) \times S \cap B\left(e_{1}, \delta_{0}\right) \times X & \rightarrow S, \\
\left(\varepsilon, z_{0}, u\right) & \rightarrow \mathcal{R}_{1}^{\varepsilon}\left(z_{0}, u\right),
\end{aligned}
$$

where $\mathcal{R}_{t}^{\varepsilon}$ is the resolving operator of problem $(3.10),(3.11), X \subset L^{2}([0,1], \mathbb{R})$ is a closed subspace and $\varepsilon_{0}>0$ and $\delta_{0}>0$ are constants. We are going to show that the measure $\mathcal{D}\left(\eta_{1}\right)$ and the function $\mathcal{R}_{1}(\cdot, \cdot)$ satisfy the conditions of Theorem 2.2 in [1] for an appropriate choice of $X$ and the constants $\varepsilon_{0}$ and $\delta_{0}$. For the reader's convenience, we recall the theorem in Section 6 (see Theorem 6.3). 
Clearly, $\mathcal{R}_{1}(\cdot, \cdot)$ is a continuous function, $\mathcal{R}_{1}^{\varepsilon}\left(z_{0}, \cdot\right)$ is analytic for any $|\varepsilon| \leq \varepsilon_{0}$ and $z_{0} \in S \cap B\left(e_{1}, \delta_{0}\right)$, and $D_{u} \mathcal{R}_{1}(\cdot, \cdot)$ is continuous.

Using Lemma 3.2, we see that for some positive constant $\nu$ the interior of the set $\mathcal{R}_{1}^{0}\left(e_{1}, B_{E_{n}}(0, \nu)\right)$ is non-empty. Denote by $P_{N}$ the orthogonal projection onto the space $\operatorname{span}\left\{g_{1}, \ldots, g_{N}\right\}$ in $L^{2}([0,1], \mathbb{R})$. The continuity of $\mathcal{R}_{1}(\cdot, \cdot)$ implies that for any $\eta>0$ there is an integer $N \geq 1$ and positive constants $\delta_{0}$ and $\varepsilon_{0}$ such that

$$
\left\|\mathcal{R}_{1}^{\varepsilon}\left(z, P_{N} u\right)-\mathcal{R}_{1}^{0}\left(e_{1}, u\right)\right\|<\eta
$$

for all $|\varepsilon| \leq \varepsilon_{0}, z \in S \cap B\left(e_{1}, \delta_{0}\right)$ and $u \in B_{E_{n}}(0, \nu)$. A standard degree theory argument implies that the interior of the set $\mathcal{R}_{1}^{\varepsilon}\left(z, P_{N}\left(B_{E_{n}}(0, \nu)\right)\right)$ is non-empty for any $|\varepsilon| \leq \varepsilon_{0}$ and $z \in S \cap B\left(e_{1}, \delta_{0}\right)$. Clearly, if (2.2) holds for the integer $N$, then conditions of Theorem 6.3 are satisfied for $X=\overline{\operatorname{span}\left\{e_{j}: b_{j} \neq 0, j \geq 1\right\}}$. Thus the function

$$
\mathcal{R}_{1 *}\left(\cdot, \mathcal{D}\left(\eta_{1}\right)\right):\left(-\varepsilon_{0}, \varepsilon_{0}\right) \times S \cap B\left(e_{1}, \delta\right) \rightarrow \mathcal{P}(S),
$$

is continuous, where $\mathcal{R}_{1 *}^{\varepsilon}\left(z, \mathcal{D}\left(\eta_{1}\right)\right)$ stands for the image of the measure $\mathcal{D}\left(\eta_{1}\right)$ under the mapping $\mathcal{R}_{1}^{\varepsilon}(z, \cdot)$ and $\mathcal{P}(S)$ is endowed with the total variation norm. This completes the proof of assertion (i).

Step 2. To prove assertion (ii), we apply Theorem 6.3 to the mapping

$$
\begin{aligned}
\mathcal{R}_{k+1}(\cdot, \cdot):\left(-\varepsilon_{0}, \varepsilon_{0}\right) \times S \times X^{k+1} & \rightarrow S, \\
(\varepsilon, z, u) & \rightarrow \mathcal{R}_{k+1}^{\varepsilon}(z, u),
\end{aligned}
$$

where $k$ is the integer in Theorem 3.5, $X$ is defined in Step 1 and $X^{k+1}$ is the set of functions $u \in L^{2}([0, k+1], \mathbb{R})$ such that $\left.u(j+\cdot)\right|_{[0,1]} \in X, j=0, \ldots, k$. Using Theorem 3.5 and the arguments of Step 1, one can show that for sufficiently small $\varepsilon_{0}>0$ and for any $z \in S$ there is a ball $B_{z}$ in a finite-dimensional subset of $X^{k+1}$ such that $\mathcal{R}_{k+1}^{\varepsilon}\left(z, B_{z}\right)$ has a non-empty interior for all $|\varepsilon|<\varepsilon_{0}$. Clearly, the other conditions of Theorem 6.3 also hold. Thus the image of the measure $\bigotimes_{j=1}^{k+1} \mathcal{D}\left(\eta_{j}\right)$ under the mapping $\mathcal{R}_{k+1}^{\varepsilon}(z, \cdot)$ (which is equal to $\mathcal{D}\left(\mathcal{U}_{k+1}^{\varepsilon}(z)\right)$ ) is absolutely continuous with respect to the Riemannian volume $m$ on $S$.

Proof of Lemma 2.5. Step 1. We write $\tau$ instead of $\tau_{\delta, \varepsilon}$. Let us show that for any $\delta>0$ there is a constant $\varepsilon_{\delta}>0$ such that

$$
\mathbb{P}_{z}\{\tau<+\infty\}=1 \text { for all }|\varepsilon|<\varepsilon_{\delta} \text { and } z \in S .
$$

Using Theorem 3.5 and Condition 2.1, one can show that for any $\delta>0$ and $z_{0} \in S$ there is a time $k=k(\delta) \geq 1$, a constant $\varepsilon_{\delta}=\varepsilon_{\delta}\left(z_{0}\right)>0$ and a neighborhood $O=O\left(z_{0}\right)$ of $z_{0}$ such that

$$
\sup _{(z, \varepsilon) \in O \times\left(-\varepsilon_{\delta}, \varepsilon_{\delta}\right)} \mathbb{P}_{z}\{\tau>k\}<1 .
$$

From the compactness of $S$ it follows that there is a constant $\varepsilon_{\delta}>0$ such that

$$
a:=\sup _{(z, \varepsilon) \in S \times\left(-\varepsilon_{\delta}, \varepsilon_{\delta}\right)} \mathbb{P}_{z}\{\tau>k\}<1 .
$$

Using the Markov property and (4.2), we obtain

$$
\mathbb{P}_{z}\{\tau>n k\}=\mathbb{E}_{z}\left(I_{\{\tau>(n-1) k\}} \mathbb{P}_{\mathcal{U}_{(n-1) k}^{\varepsilon}(\cdot)}\{\tau>k\}\right) \leq a \mathbb{P}_{z}\{\tau>(n-1) k\} .
$$


Thus

$$
\mathbb{P}_{z}\{\tau>n k\} \leq a^{n}
$$

This proves (4.1).

Step 2. Using (4.1) and (4.3), we obtain for sufficiently small $\alpha>0$

$$
\begin{aligned}
\mathbb{E}_{z} e^{\alpha \tau} & \leq 1+\sum_{n=0}^{\infty} \mathbb{E}_{z}\left(e^{\alpha \tau} I_{\{n k<\tau \leq(n+1) k\}}\right) \leq 1+\sum_{n=0}^{\infty} e^{\alpha(n+1) k} \mathbb{P}_{z}\{\tau>n k\} \\
& \leq 1+\sum_{n=0}^{\infty} e^{\alpha(n+1) k} a^{n}=1+\frac{e^{\alpha k}}{1-e^{\alpha k} a}
\end{aligned}
$$

REMARK 4.1. An estimate similar to (2.4) holds for the Markov chain $\left(y_{n}, y_{n}^{\prime}\right)$ constructed in Step 2 of the proof of Theorem 2.3. Namely, let $T$ be the stopping time defined by (2.6). Let us show that (2.7) holds.

Indeed, it follows from the above proof that inequality (2.7) will be established if we show that for any $\delta>0$ and $z_{0}, z_{0}^{\prime} \in S$ there is a time $l=l\left(\delta, z_{0}, z_{0}^{\prime}\right) \geq 1$, a constant $\varepsilon_{\delta}=\varepsilon_{\delta}\left(z_{0}, z_{0}^{\prime}\right)>0$ and a neighborhood $O=O\left(z_{0}, z_{0}^{\prime}\right)$ of the point $\left(z_{0}, z_{0}^{\prime}\right)$ such that

$$
\sup _{\left(z, z^{\prime}, \varepsilon\right) \in O \times\left(-\varepsilon_{\delta}, \varepsilon_{\delta}\right)} \mathbb{P}_{\left(z, z^{\prime}\right)}\{T>l\}<1 .
$$

The case $z_{0}=z_{0}^{\prime}$ follows from the definition of the sequence $\left(y_{n}, y_{n}^{\prime}\right)$ and (4.2), and the case $z_{0}, z_{0}^{\prime} \in B\left(e_{1}, \delta\right)$ is clear. Thus it suffices to prove (4.4) in the case $z_{0} \neq z_{0}^{\prime}$ and $z_{0} \notin B\left(e_{1}, \delta\right)$. Let $(\Omega, \mathcal{F}, \mathbb{P})$ be the underlying probability space. Define the event

$$
\Omega_{1}:=\left\{\omega \in \Omega: y_{n}=y_{n}^{\prime} \text { for some } n=1, \ldots, k\right\} \in \mathcal{F},
$$

where $k \geq 1$ is the integer in Theorem 3.5. Let $\Omega_{2}:=\Omega \backslash \Omega_{1}$. It follows from the definition of $\left(y_{n}, y_{n}^{\prime}\right)$ that

$$
y_{k}=y_{k}^{\prime} \text { for any } \omega \in \Omega_{1} \text {. }
$$

Using again the definition of $\left(y_{n}, y_{n}^{\prime}\right)$ and (4.2), we get

$$
\sup _{\left(z, z^{\prime}, \varepsilon\right) \in O^{\prime} \times\left(-\varepsilon_{\delta}^{\prime}, \varepsilon_{\delta}^{\prime}\right)} \mathbb{P}_{\left(z, z^{\prime}\right)}\left\{\tau>2 k \mid \Omega_{1}\right\}<1,
$$

where $\varepsilon_{\delta}^{\prime}=\varepsilon_{\delta}^{\prime}\left(z_{0}, z_{0}^{\prime}\right)>0$ and $O^{\prime}=O^{\prime}\left(z_{0}, z_{0}^{\prime}\right)$ is a neighborhood of the point $\left(z_{0}, z_{0}^{\prime}\right)$. On the other hand, by Theorem 3.5, Condition 2.1 and the construction of $\left(y_{n}, y_{n}^{\prime}\right)$, we have

$$
\sup _{\left(z, z^{\prime}, \varepsilon\right) \in O^{\prime \prime} \times\left(-\varepsilon_{\delta}^{\prime \prime}, \varepsilon_{\delta}^{\prime \prime}\right)} \mathbb{P}_{\left(z, z^{\prime}\right)}\left\{\tau>k \mid \Omega_{2}\right\}<1
$$

for some $\varepsilon_{\delta}^{\prime \prime}=\varepsilon_{\delta}^{\prime \prime}\left(z_{0}, z_{0}^{\prime}\right)>0$ and $O^{\prime \prime}=O^{\prime \prime}\left(z_{0}, z_{0}^{\prime}\right)$. Combining (4.5) and (4.6), we get (4.4). 


\section{Application}

In this section, we apply Theorem 2.3 to the Galerkin approximations of the Schrödinger equation. For any integer $k \geq 2$, denote by $Q_{k}$ the vector space of all polynomials of degree $k$ with real coefficients. Let $\lambda$ be the Lebesgue measure on $Q_{k}$.

Consider the problem

$$
\begin{aligned}
i \frac{\partial z}{\partial t} & =-z^{\prime \prime}+\beta(t) V(x) z+\varepsilon|z|^{\sigma} z, \quad x \in(0,1), \\
z(t, 0) & =z(t, 1)=0, \\
z(0, x) & =z_{0}(x),
\end{aligned}
$$

where $\sigma>0$ and $\varepsilon$ are constants, $\beta(t)$ is a random process of the form (1.3) and $V \in Q_{k}$. Denote by $\left\{e_{j}\right\}_{j \in \mathbb{N}}$ the set of normalized eigenfunctions of the Dirichlet Laplacian. For any $n \in \mathbb{N}$, let $H_{n}:=\operatorname{span}\left\{e_{1}, \ldots, e_{n}\right\}$ and let $P_{n}$ be the orthogonal projection onto $H_{n}$ in $L^{2}([0,1])$. The Galerkin approximation of order $n$ of $(5.1)$ has the form

$$
i \frac{\partial z}{\partial t}=-z^{\prime \prime}+\beta(t) P_{n}(V(x) z)+\varepsilon P_{n}\left(|z|^{\sigma} z\right) .
$$

Clearly, (5.4) can be rewritten in the form (1.1). Denote by $\langle\cdot, \cdot\rangle$ the scalar product in $L^{2}([0,1])$ and by $S$ the unit sphere in $H_{n}$.

Theorem 5.1. Suppose that Condition 2.1 is satisfied. Then for $\lambda$-almost all $V \in Q_{k}$ there is an integer $N \geq 1$ and a constant $\varepsilon_{0}>0$ such that, if (2.2) holds, then problem (5.4), (1.3) has a unique stationary measure $\mu \in \mathcal{P}(S)$ for $|\varepsilon|<\varepsilon_{0}$. Moreover, $\mu$ is absolutely continuous with respect to the Riemannian volume on $S$ and for any initial measure $\nu \in \mathcal{P}(S)$ inequality (2.3) holds.

Proof. It suffices to note that $\left\langle x^{2} e_{1}, e_{j}\right\rangle \neq 0$ for $j=1, \ldots, n$, and thus the set of polynomials $V \in Q_{k}$ with $\left\langle V e_{1}, e_{i}\right\rangle=0$ for some $i=1, \ldots, n$ has a zero $\lambda$-measure. It remains to apply Theorem 2.3 .

\section{Appendix}

Here we recall a result on the finite-dimensional transformations of measures. Let $X$ be a separable Hilbert space with the norm $\|\cdot\|_{X}$. We deal with measures $\mu \in \mathcal{P}(X)$ satisfying the following condition.

Condition 6.1. The measure $\mu \in \mathcal{P}(X)$ has a finite second moment

$$
\int_{X}\|x\|_{X}^{2} \mu(\mathrm{d} x)<\infty .
$$

Moreover, there is an orthonormal basis $\left\{g_{j}\right\} \subset X$ such that

$$
\mu=\bigotimes_{j=1}^{\infty} \mu_{j}
$$

where $\mu_{j}$ is the projection of $\mu$ to the space $X_{j}$ generated by $g_{j}$ and $\otimes$ denotes the tensor product of measures. Finally, for any $j \in \mathbb{N}$, the measure $\mu_{j}$ possesses a continuous density with respect to the Lebesgue measure on $X_{j}$. 
EXAMPle 6.2. Let $\eta$ be a random variable satisfying Condition 2.1. Then choosing $X:=\overline{\operatorname{span}\left\{e_{j}: b_{j} \neq 0, j \geq 1\right\}}$, it is easy to see that Condition 6.1 is satisfied for the measure $\mathcal{D}(\eta) \in \mathcal{P}(X)$.

Let $H$ be a metric space and $M$ be a finite-dimensional analytic Riemannian manifold.

THEOREM 6.3. Let $f: H \times X \rightarrow M$ be a continuous function such that $f(u, \cdot)$ is analytic for any $u \in H$ and the derivative $D_{x} f(u, x)$ is continuous with respect to $(u, x)$. Suppose that, for any $u \in H$, there is a ball $B_{u}$ in a finite-dimensional subspace $X_{u} \subset X$ such that the interior of the set $f\left(u, B_{u}\right)$ is non-empty. Then for any measure $\mu \in \mathcal{P}(X)$ satisfying Condition 6.1, we have:

(i) For any $u_{0} \in H$, the measure $f_{*}\left(u_{0}, \mu\right)$ is absolutely continuous with respect to the Riemannian volume on $M$, where $f_{*}\left(u_{0}, \mu\right)$ is the image of the measure $\mu$ under the mapping $f\left(u_{0}, \cdot\right): X \rightarrow M$.

(ii) The function $f_{*}\left(u_{0}, \mu\right)$ from $H$ to the space $\mathcal{P}(M)$ endowed with the total variation norm is continuous.

See [1] for the proof of this theorem in the case of finite-dimensional vector space $M$. The result in the case of a Riemannian manifold is deduced from the case of finite-dimensional vector space.

Note that the main result of the paper could be stated under more general assumptions over the distribution of the random variable $\eta_{1}$ that are adapted to a measure transformation theorem from [3]. Our choice is explained by the simplicity of the conditions of Theorem 6.3.

\section{References}

[1] A. Agrachev, S. Kuksin, A. Sarychev, A. Shirikyan, On finite-dimensional projections of distributions for solutions of randomly forced 2D Navier-Stokes equations, Annales de l'IHP, Prob. Stat., 43, 399-415, 2007.

[2] A. Agrachev, A. Sarychev, Navier-Stokes equations: controllability by means of low modes forcing, J. Math. Fluid Mech., 7, 108-152, 2005.

[3] D. Alexandrova, V. Bogachev, A. Pilipenko, On the convergence in the variation norm for the images of measures under differentiable mappings, Sbornik: Mathematics, 190, 9, 1229-1245, 1999.

[4] F. Albertini, D. D'Alessandro, Notions of controllability for bilinear multilevel quantum systems, IEEE Transactions on Automatic Control, 48, 8, 1399-1403, 2003.

[5] L. Arnold, Random Dynamical Systems, Springer-Verlag, Berlin, 1998.

[6] L. Arnold, W. Kliemann, On unique ergodicity for degenerate diffusions, Stochastics 21, 41-61, 1987.

[7] K. Beauchard, Local Controllability of a 1-D Schrödinger equation, J. Math. Pures et Appl., 84, 7, 851-956, 2005.

[8] K. Beauchard, J.-M. Coron, M. Mirrahimi, P. Rouchon, Implicit Lyapunov control of finite dimensional Schrödinger equations, System and Control Letters, 56, 5, 388-395, 2007.

[9] W. E, J. Mattingly, Ergodicity for the Navier-Stokes equation with degenerate random forcing: finite-dimensional approximation, Comm. Pure Appl. Math., 54, 11, 1386-1402, 2001.

[10] R. Z. Hasminskii, Stochastic Stability of Differential Equations, Sijthoff \& Noordhoff, Alphen aan den Rijn, 1980.

[11] S. Kuksin, A. Shirikyan, A coupling approach to randomly forced nonlinear PDE's. I, Comm. Math. Phys., 221, 2, 351-366, 2001.

[12] T. Lindvall, Lectures on the Coupling Method, Wiley, New York, 1992.

[13] S. Meyn, R. Tweedie, Markov Chains and Stochastic Stability, Springer, Berlin, 1993.

[14] S. Meyn, R. Tweedie, Stability of Markovian processes I-III, Adv. Appl. Proba., 24, 542-574, 25, 487-548, 1993. 
[15] M. Romito, Ergodicity of the finite dimensional approximation of the 3D Navier-Stokes equations forced by a degenerate noise, J. Statist. Physics, 114, 155-177, 2004.

[16] A. Shirikyan, Approximate controllability of three-dimensional Navier-Stokes equations, Comm. Math. Phys., 266, 1, 123-151, 2006.

[17] A. Yu. Veretennikov, Estimates of the mixing rate for stochastic equations, Theory Probab. Appl., 32, 2, 273-281, 1987.

[18] A. Yu Veretennikov, The mixing rate and the averaging principle for hypoelliptic stochastic differential equations, translation in Math. USSR-Izv., 33, 2, 221-231, 1989.

Laboratoire de Mathématiques, Université de Paris-Sud Xi Bâtiment 425, 91405 Orsay Cedex, France

E-mail address: Vahagn.Nersesyan@math.u-psud.fr 